CERN-TH/95-264

UWThPh-32-1995

hep-th/9510083

\title{
Topologically Nontrivial Field Configurations in Noncommutative Geometry
}

\author{
H. Grosse円 \\ Institut for Theoretical Physics, University of Vienna, \\ Boltzmanngasse 5, A-1090 Vienna, Austria \\ C. Klimčík \\ Theory Division CERN \\ CH-1211 Geneva 23, Switzerland \\ P. Prešnajder \\ Department of Theoretical Physics, Comenius University \\ Mlynská dolina, SK-84215 Bratislava, Slovakia
}

\begin{abstract}
In the framework of noncommutative geometry we describe spinor fields with nonvanishing winding number on a truncated (fuzzy) sphere. The corresponding field theory actions conserve all basic symmetries of the standard commutative version (space isometries and global chiral symmetry), but due to the noncommutativity of the space the fields are regularized and they contain only finite number of modes.
\end{abstract}

CERN-TH/95-264

October 1995

\footnotetext{
${ }^{1}$ Part of the Project P8916-PHY of the 'Fonds zur Förderung der wissentschaftlichen Forschung in Österreich'.
} 


\section{Introduction}

The basic notions of the noncommutative geometry were developed in [1, 2], and, in the form of the matrix geometry, in [3, 4. The essence of this approach consists in reformulating first the geometry in terms of commutative algebras and modules of smooth functions, and then generalizing them to their noncommutative analogues.

In standard field theory, to any point $x$ of some space(-time) manifold $M$ the values of various fields are assigned:

$$
x \in M \longrightarrow \Phi(x), A(x), \ldots,
$$

as sections of some bundles over $M$, e.g. the line bundle of functions, or the spinor bundle, etc. The smooth functions on $M$ form a commutative algebra $\mathcal{A}=\mathcal{F}(\mathcal{M})$ with respect to the standard pointwise product: $(f g)(x)=$ $f(x) g(x), x \in M$. The bundle of smooth spinor fields $\mathcal{S}(M)$ on $M$ is an $\mathcal{A}$ module with respect to the multiplication by smooth functions, which simply means that any spinor can by multiplied by a scalar field. In the same way the linear spaces of gauge and other fields are $\mathcal{A}$-modules. If there exists a sequence of deformations of the commutative algebra $\mathcal{A}$ of the smooth functions on the manifold $M$, such that the deformed algebras are finite dimensional, we may attempt to formulate a deformed field theory which would possess just a finite number of degrees of freedom. Needless to say, this may be a promising avenue towards a suitable regularization of ill-defined field theoretical path integrals.

Having the finite deformations of the algebra of scalar fields (= functions on the manifold $M$ ) a further step has to be made in order to obtain a physically useful regularization. It consists of building up deformed spinor bundles, gauge fields, etc., which would be also just the finite-dimensional vector spaces and at the same time modules of the deformed algebra $\mathcal{A}$ of the scalar fields. For a fixed deformation of $\mathcal{A}$ there may be many inequivalent deformations of the corresponding modules. Therefore we would like to have a guiding principle that would select the deformations which could legitimately be called the noncommutative spinor bundles or spaces of noncommutative connections. In the general case of an arbitrary compact Riemannian manifold we are still missing this principle; however, for practical applications in Euclidean field theories it is enough to understand the noncommutative deformations of the spheres $S^{n}$. Then the guiding principle reads: build up 
the noncommutative modules, which would be representation spaces of the symmetry group that rotates the sphere $S^{n}$.

It is also natural to require that the regularized field theory path integral respects the rotational symmetry. This significantly narrows the room for the possible deformations. Further restrictions come from a claim that some other desirable field theory symmetries are preserved in the deformed level. Amazingly, our experience so far shows that all relevant symmetries can be incorporated while preserving the finiteness of the number of degrees of freedom. To our knowledge this is an unusual (and very favourable) property; for instance the fashionable lattice regularization does not enjoy it.

In our previous investigations [5, 6], we dealt with the two-dimensional field theories, i.e. the manifold $M$ was the sphere $S^{2}$. The real scalar field on a truncated (= deformed) sphere was considered in [5] and, recently, we found a proper supersymmetric extension of this formalism [6]. In particular, the latter accounts for the description of the (chiral) spinor fields with vanishing topological charge and gives a manifestly supersymmetric regularization of two-dimensional supersymmetric theories. Historically, the truncated two-sphere] was introduced in [0], where the deformed algebra of functions emerged upon geometric quantization of the (symplectic) volume form on the sphere. Later the concept was rediscovered in [8, 9, 10]. The first attempts to construct a field theory on the truncated sphere were undertaken in [9, 11]; in [5, 6] we have included the details of the perturbation expansion for the deformed quantum scalar field, the construction of the deformed chiral spinors and the regularization of the supersymmetric theories.

In this article, we continue these investigations by constructing the deformed topologically nontrivial spinor bundles needed for the inclusion of the monopoles. As was argued in [12], the correct infinite volume limit (which means that the radius of the sphere approaches infinity) requires the consideration of the monopole configurations of the gauge fields interacting with the spinors. Hence, having in mind our ultimate goal of the physical applicability of the construction, we have to incorporate the spinor bundles with a nontrivial winding number. Remarkably, this can be done already at the 'kinematical' level of the configuration space of the deformed field theory as opposed to the case of the lattice regularization, where the topologically

\footnotetext{
${ }^{2}$ Also referred to as 'fuzzy', 'noncommutative' or 'quantum' sphere in the literature 迥, 8, 9].
} 
nontrivial configurations emerge only dynamically [13].

The plan of the paper is as follows: All basic notions we need on complex scalar and spinor fields in the standard (commutative) case are summarized in Section 2. In Section 3 we first describe the topologically nontrivial configurations of a complex scalar field on a standard sphere in a more algebraic language, and then we generalize them to the noncommutative situation. In Section 4 we extend our approach to the topologically nontrivial spinor field configurations on the noncommutative sphere and write down a chirally symmetric field theory action. Section 5 contains concluding remarks.

\section{Topologically nontrivial fields on the sphere}

Here we briefly describe the topologically nontrivial configurations of complex scalar and spinor fields on the standard two-sphere. The embedding $S^{2} \hookrightarrow \mathbf{R}^{3}$ is specified by the Cartesian coordinates

$$
x_{1}=r \cos \varphi \sin \theta, x_{2}=r \sin \varphi \sin \theta, x_{3}=r \cos \theta,
$$

where $r>0$ is fixed, $0 \leq \theta \leq \pi,-\pi \leq \varphi \leq+\pi$.

The complex scalar field $\Phi$ on the upper hemisphere $V_{+}\left(S^{2}\right.$ without south pole $\theta \neq \pi)$ is a function of the variables

$$
\chi_{1}^{\prime}=r^{1 / 2} \cos \frac{\theta}{2}, \chi_{2}^{\prime}=-r^{1 / 2} \sin \frac{\theta}{2} e^{i \varphi},
$$

which are well defined on $V_{+}$:

$$
\Phi^{\prime}=\Phi^{\prime}\left(\chi^{\prime}, \chi^{\prime *}\right)=\sum a_{m_{1} m_{2} n_{1} n_{2}}^{\prime} \chi_{1}^{\prime * m_{1}} \chi_{2}^{\prime * m_{2}} \chi_{1}^{\prime n_{1}} \chi_{2}^{\prime n_{2}} .
$$

The monomials in this expansion are characterized by their phase on the equator $(\theta=\pi / 2)$

$$
e^{i\left(m_{2}-n_{2}\right) \varphi}=\left.\chi_{1}^{\prime * m_{1}} \chi_{2}^{\prime * m_{2}} \chi_{1}^{\prime n_{1}} \chi_{2}^{\prime n_{2}}\right|_{\theta=\pi / 2} .
$$

In the same way, the complex scalar field $\Phi$ on the lower hemisphere $V_{-}$ $\left(S^{2}\right.$ without north pole $\theta \neq 0$ ) is a function of the variables

$$
\chi^{\prime \prime}{ }_{1}=r^{1 / 2} \cos \frac{\theta}{2} e^{-i \varphi}, \chi^{\prime \prime}{ }_{2}=-r^{1 / 2} \sin \frac{\theta}{2} .
$$


Thus,

$$
\Phi^{\prime \prime}=\Phi^{\prime \prime}\left(\chi^{\prime \prime}, \chi^{\prime \prime *}\right)=\sum a_{m_{1} m_{2} n_{1} n_{2}}^{\prime \prime} \chi_{1}^{\prime \prime * m_{1}} \chi_{2}^{\prime \prime * m_{2}} \chi_{1}^{\prime \prime n_{1}} \chi_{2}^{\prime \prime n_{2}}
$$

Now, the monomials in this expansion have the following phase on the equator $(\theta=\pi / 2)$

$$
e^{i\left(n_{1}-m_{1}\right) \varphi}=\left.\chi_{1}^{\prime \prime * m_{1}} \chi_{2}^{\prime \prime * m_{2}} \chi_{1}^{\prime \prime n_{1}} \chi_{2}^{\prime \prime n_{2}}\right|_{\theta=\pi / 2} .
$$

As $\tilde{\mathcal{H}}_{k}, k \in \frac{1}{2} \mathbf{Z}$, we denote the line bundle of sections with the same expansion coefficients as in eqs. (3) and (5):

$$
\begin{gathered}
\Phi^{\prime}=\Phi\left(\chi^{\prime}, \chi^{* *}\right)=\sum a_{m_{1} m_{2} n_{1} n_{2}} \chi_{1}^{\prime * m_{1}} \chi_{2}^{\prime * m_{2}} \chi_{1}^{\prime n_{1}} \chi_{2}^{\prime n_{2}} . \\
\Phi^{\prime \prime}=\Phi\left(\chi^{\prime \prime}, \chi^{\prime \prime *}\right)=\sum a_{m_{1} m_{2} n_{1} n_{2}} \chi_{1}^{\prime \prime * m_{1}} \chi_{2}^{\prime \prime * m_{2}} \chi_{1}^{\prime \prime n_{1}} \chi_{2}^{\prime \prime n_{2}},
\end{gathered}
$$

and with $k=\frac{1}{2}\left(m_{1}+m_{2}-n_{1}-n_{2}\right)$ fixed. On $V_{+} \cap V_{-}$they are related by the singular gauge transformation

$$
\Phi^{\prime}=e^{i \kappa \varphi} \Phi^{\prime \prime}
$$

where $\kappa=2 k$ is the so-called topological winding number. Obviously, $\mathcal{A}=\tilde{\mathcal{H}}_{0}$ is an algebra, and $\tilde{\mathcal{H}}_{k}$ are $\mathcal{A}$-modules (with respect to the usual multiplication by functions from $\mathcal{A}$ ).

The presence of the gauge transformation (7) requires the use of the covariant derivatives:

$$
\begin{aligned}
D_{\mu}^{\prime} & =i \partial_{\mu}^{\prime}+A_{\mu}^{\prime}, \quad \text { on } V_{+}, \\
D_{\mu}^{\prime \prime} & =i \partial_{\mu}^{\prime \prime}+A_{\mu}^{\prime \prime} \text { on } V_{-} .
\end{aligned}
$$

Here we introduced the topological ( $\kappa$-monopole) fields

$$
\begin{aligned}
& A_{\mu}^{\prime}=i \kappa \chi^{\prime+} \partial_{\mu}^{\prime} \chi^{\prime}, \text { on } V_{+}, \\
& A_{\mu}^{\prime \prime}=i \kappa \chi^{\prime \prime} \partial_{\mu}^{\prime \prime} \chi^{\prime \prime}, \text { on } V_{-} .
\end{aligned}
$$

On $V_{+} \cap V_{-}$they are related by the singular gauge transformation (7):

$$
A_{\mu}^{\prime}=A_{\mu}^{\prime \prime}-i h \partial_{\mu} h^{-1}, h=e^{i \kappa \varphi} .
$$

Note: We would like to stress that the presence of the topological $\kappa$ monopole field is dictated by the nontrivial topology, and not by the dynamics 
of a system in question. The dynamical gauge field $\mathcal{A}_{\mu}=\mathcal{A}_{\mu}(x)$ is globally defined on $S^{2}$, and it could be added to the topological one. In what follows, we shall not consider this possibility.

The action for the complex scalar field on $S^{2}$ can be written in the form

$$
S\left[\Phi, \Phi^{*}\right]=\frac{1}{2 \pi r} \int d^{3} x \delta\left(x^{2}-r^{2}\right)\left[\Phi^{*} D_{\mu}^{2} \Phi+V\left(\Phi^{*} \Phi\right)\right]
$$

where $V($.$) is a polynomial bounded from below.$

In the same manner, the spinor field $\Psi$ we define separately on the upper and lower hemisphere:

$$
\begin{aligned}
& \Psi^{\prime}=\left(\begin{array}{l}
\Psi_{1}^{\prime} \\
\Psi_{2}^{\prime}
\end{array}\right), \text { on } V_{+}, \\
& \Psi^{\prime \prime}=\left(\begin{array}{l}
\Psi_{1}^{\prime \prime} \\
\Psi_{2}^{\prime \prime}
\end{array}\right), \text { on } V_{+} .
\end{aligned}
$$

As $\tilde{\mathcal{S}}_{k}$ we denote the bundle of spinor fields, which have their components from $\tilde{\mathcal{H}}_{k}$ :

$$
\begin{gathered}
\Psi_{\alpha}^{\prime}=\Psi_{\alpha}\left(\chi^{\prime}, \chi^{\prime *}\right)=\sum a_{m_{1} m_{2} n_{1} n_{2}}^{\alpha} \chi_{1}^{\prime * m_{1}} \chi_{2}^{\prime * m_{2}} \chi_{1}^{\prime n_{1}} \chi_{2}^{\prime n_{2}} . \\
\Psi_{\alpha}^{\prime \prime}=\Phi_{\alpha}\left(\chi^{\prime \prime}, \chi^{\prime \prime *}\right)=\sum a_{m_{1} m_{2} n_{1} n_{2}}^{\alpha} \chi_{1}^{\prime \prime * m_{1}} \chi_{2}^{\prime \prime * m_{2}} \chi_{1}^{\prime \prime n_{1}} \chi_{2}^{\prime \prime n_{2}},
\end{gathered}
$$

where $k=\frac{1}{2}\left(m_{1}+m_{2}-n_{1}-n_{2}\right)$ is fixed. Obviously, $\tilde{\mathcal{S}}_{k}$ are $\mathcal{A}$-modules.

Alternatively, we can write the elements of the $\tilde{\mathcal{S}}_{k}$ in the form

$$
\begin{gathered}
\Psi^{\prime}=f\left(\chi^{\prime}, \chi^{\prime *}\right)\left(\begin{array}{c}
\chi_{1}^{\prime} \\
\chi_{2}^{\prime}
\end{array}\right)+g\left(\chi^{\prime}, \chi^{\prime *}\right)\left(\begin{array}{c}
\chi_{2}^{\prime *} \\
-\chi_{1}^{\prime *}
\end{array}\right), \text { on } V_{+}, \\
\Psi^{\prime \prime}=f\left(\chi^{\prime \prime}, \chi^{\prime \prime *}\right)\left(\begin{array}{c}
\chi^{\prime \prime}{ }_{1} \\
\chi_{2}^{\prime \prime}
\end{array}\right)+g\left(\chi^{\prime \prime}, \chi^{\prime \prime *}\right)\left(\begin{array}{c}
\chi_{2}^{\prime \prime *} \\
-\chi_{1}^{\prime \prime *}
\end{array}\right) \text {, on } V_{-} .
\end{gathered}
$$

The advantage of this form lies in the fact that both the $f$ and $g$ parts of this decomposition are separately eigenfunctions of the chirality operator

$$
\Gamma=\frac{1}{r} \sigma_{i} x_{i}, \sigma_{i}-\text { Pauli matrices }
$$


with the eigenvalues +1 and -1 respectively:

$$
\begin{gathered}
\Gamma \Psi^{\prime}=f\left(\chi^{\prime}, \chi^{\prime *}\right)\left(\begin{array}{c}
\chi_{1}^{\prime} \\
\chi_{2}^{\prime}
\end{array}\right)-g\left(\chi^{\prime}, \chi^{\prime *}\right)\left(\begin{array}{c}
\chi_{2}^{\prime *} \\
-\chi_{1}^{\prime *}
\end{array}\right), \\
\Gamma \Psi^{\prime \prime}=f\left(\chi^{\prime \prime}, \chi^{\prime \prime *}\right)\left(\begin{array}{c}
\chi_{1}^{\prime \prime} \\
\chi_{2}^{\prime \prime}
\end{array}\right)-g\left(\chi^{\prime \prime}, \chi^{\prime \prime *}\right)\left(\begin{array}{c}
\chi_{2}^{\prime \prime *} \\
-\chi_{1}^{\prime \prime *}
\end{array}\right) .
\end{gathered}
$$

The action of the (Dirac) spinor field can be written as

$$
S\left[\Psi, \Psi^{*}\right]=\frac{1}{2 \pi r} \int d^{3} x \delta\left(x^{2}-r^{2}\right)[\bar{\Psi} D \Psi+W(\bar{\Psi}, \Psi)]
$$

where $\bar{\Psi}=\Psi^{+} \sigma^{2}, W(.,$.$) is a gauge- and chiral-invariant potential describing$ the selfinteraction of the spinor field, and $D$ is a Dirac operator defined as

$$
\begin{aligned}
D^{\prime}=r\left[i \sigma^{\prime \mu}\left(\partial_{\mu}^{\prime}+A_{\mu}^{\prime}\right)+\frac{1}{r}\right], & \text { on } V_{+}, \\
D^{\prime \prime}=r\left[i \sigma^{\prime \prime \mu}\left(\partial_{\mu}^{\prime \prime}+A_{\mu}^{\prime \prime}\right)+\frac{1}{r}\right], & \text { on } V_{-},
\end{aligned}
$$

Here $\sigma^{\mu}=\sigma_{i} e_{i}^{\mu}$ ( $e_{i}^{\mu}$ denote corresponding zwei-beins). Only the topological ( $\kappa$-monopole) gauge field enters into $D$, and not a dynamical one. The Dirac operator anticommutes with the chirality operator,

$$
D \Gamma+\Gamma D=0
$$

This guarantees the chiral invariance of the action (16).

In the next two sections we first rewrite all relevant formulas in more algebraic terms, and then we introduce their truncated noncommutative analogues.

\section{Complex scalar field}

We start with the Hopf fibration obtained from the mapping $\mathbf{C}^{2} \rightarrow \mathbf{R}^{3}$ defined by

$$
\chi=\left(\begin{array}{l}
\chi_{1} \\
\chi_{2}
\end{array}\right) \longrightarrow x=\left(x_{1}, x_{2}, x_{3}\right)
$$


where

$$
x_{i}=\chi^{+} \sigma_{i} \chi, \quad i=1,2,3 .
$$

The restriction $\chi^{+} \chi=r>0$ implies $x_{i}^{2}=r^{2}$, i.e. we obtain the fibration $S^{3} \rightarrow S^{2}$ of a three-sphere in $\mathbf{C}^{2}$ with the radius $\sqrt{r}$ onto the two-sphere in $\mathbf{R}^{3}$ with the radius $r$. Since $x_{i}$ do not change under the transformation

$$
\chi \rightarrow e^{\frac{i}{2} \psi} \chi, \chi^{+} \rightarrow e^{-\frac{i}{2} \psi} \chi^{+},
$$

we see that the fibre is $U(1)$.

As $\mathcal{H}_{k}, k \in \frac{1}{2} \mathbf{Z}$, we denote the linear space of functions in $\mathbf{C}^{2}$ (or in $S^{3}$ after the restriction) of the form

$$
\Phi=\Phi\left(\chi, \chi^{*}\right)=\sum a_{m_{1} m_{2} n_{1} n_{2}} \chi_{1}^{* m_{1}} \chi_{2}^{* m_{2}} \chi_{1}^{n_{1}} \chi_{2}^{n_{2}},
$$

with $k=\frac{1}{2}\left(m_{1}+m_{2}-n_{1}-n_{2}\right)$ fixed (* denotes complex conjugation). Under (19) the functions from $\mathcal{H}_{k}$ transform as

$$
\Phi \rightarrow e^{-i k \psi} \Phi .
$$

They are eigenfunctions of the operator

$$
K_{0}=\frac{1}{2}\left[\chi_{\alpha}^{*} \partial_{\chi_{\alpha}^{*}}-\chi_{\alpha} \partial_{\chi_{\alpha}}\right]
$$

with the eigenvalue $k$ :

$$
K_{0} \Phi=k \Phi, \Phi \in \mathcal{H}_{k} .
$$

We have an involutive gradation

$$
\mathcal{H}_{k}^{*}=\mathcal{H}_{-k}, \mathcal{H}_{k} \mathcal{H}_{l} \subset \mathcal{H}_{k+l}
$$

with respect to the point-wise multiplication of functions

$$
\left(\Phi_{1}, \Phi_{2}\right)\left(\chi, \chi^{*}\right)=\Phi_{1}\left(\chi, \chi^{*}\right) \Phi_{2}\left(\chi, \chi^{*}\right) .
$$

The space $\mathcal{A}=\mathcal{H}_{0}$ endowed with the product (22) is a commutative algebra, which is isomorphic to the algebra of all polynomials in the variables $x_{i}, i=$ $1,2,3$. Obviously, all $\mathcal{H}_{k}$ are $\mathcal{A}$-modules.

The differential operators

$$
J_{k}=\frac{i}{2}\left[\chi_{\beta}^{*} \sigma_{\alpha \beta}^{* k} \partial_{\chi_{\alpha}^{*}}-\chi_{\beta} \sigma_{\alpha \beta}^{k} \partial_{\chi_{\alpha}}\right], k=1,2,3,
$$


map $\mathcal{H}_{k}$ to $\mathcal{H}_{k}$ and satisfy in $\mathcal{H}_{k}$ the $s u(2)$-algebra relations

$$
\left[J_{i}, J_{j}\right]=i \varepsilon_{i j k} J_{k}
$$

The formulas

$$
J_{j} \chi_{\beta}=\frac{1}{2 i} \sigma_{\alpha \beta}^{j} \chi_{\alpha}, J_{j} \chi_{\beta}^{*}=-\frac{1}{2 i} \sigma_{\alpha \beta}^{* j} \chi_{\alpha}^{*}
$$

guarantee that $\chi$ and $\chi^{*}$ transform like spinors under transformations generated by (24), and consequently $x$ transforms like a vector in $\mathbf{R}^{3}$. Moreover, the function $C(x)=x_{i}^{2}=\left(\chi^{+} \sigma_{i} \chi\right)^{2}$ satisfies

$$
J_{i} C(x)=0, i=1,2,3,
$$

i.e. $C(x)$ is an invariant function as expected.

Besides these operators we introduce operators $K_{+}$and $K_{-}$defined as

$$
K_{+} \Phi=i \varepsilon_{\alpha \beta} \chi_{\alpha}^{*}\left(\partial_{\chi_{\beta}} \Phi\right), K_{-} \Phi=i \varepsilon_{\alpha \beta}\left(\partial_{\chi_{\alpha}^{*}} \Phi\right) \chi_{\beta}
$$

They map $\mathcal{H}_{k}$ to $\mathcal{H}_{k+1}$ and $\mathcal{H}_{k-1}$ respectively. The operators $K_{ \pm}$and $K_{0}$ satisfy $s u(2)$ algebra relations

$$
\left[K_{0}, K_{ \pm}\right]= \pm K_{ \pm},\left[K_{+}, K_{-}\right]=2 K_{0} .
$$

Only products $K_{ \pm} K_{\mp}$ act in $\mathcal{H}_{k}$, and $K_{0}$ takes there the constant value $k$. The operators $K_{0}, K_{ \pm}$commute with $J_{i}, i=1,2,3$, but they are not independent as the corresponding Casimir operators are equal:

$$
J_{i}^{2}=K_{0}^{2}+\frac{1}{2}\left(K_{+} K_{-}+K_{-} K_{+}\right) \text {. }
$$

To any $\Phi \in \mathcal{A}$ we assign the standard integral over $S^{2}$

$$
I_{\infty}[\Phi]=\frac{1}{2 \pi r} \int d^{3} x \delta\left(x_{i}^{2}-r^{2}\right) \Phi(x)
$$

This allows the introduction of the scalar product on $\mathcal{H}_{k}$ as follows:

$$
\left(\Phi_{1} \Phi_{2}\right)_{k}=I_{\infty}\left[\Phi_{1}^{*} \Phi_{2}\right]
$$

\footnotetext{
${ }^{3}$ It is important to note that the operators $J_{k}$ and $K_{0}$ do commute with the restriction $\chi^{+} \chi=r$, so they naturally act on the algebra of functions on $S^{3}$.
} 
We identify the complex scalar field $\Phi$ with the topological charge $\kappa$ f with the elements of $\mathcal{H}_{k}$. The corresponding field action is given as

$$
S\left[\Phi, \Phi^{*}\right]=I_{\infty}\left[\frac{1}{2} \Phi^{*}\left(K_{+} K_{-}+K_{-} K_{+}\right) \Phi+V\left(\Phi^{*} \Phi\right)\right]
$$

where $V($.$) is a polynomial bounded from below. According to eq. (27) the$ differential operator $\frac{1}{2}\left(K_{+} K_{-}+K_{-} K_{+}\right)$can be rewritten in $\mathcal{H}_{k}$ as follows:

$$
\frac{1}{2}\left(K_{+} K_{-}+K_{-} K_{+}\right)=J_{i}^{2}-k^{2} .
$$

We stress that formula (30) for the action is equivalent to (11).

We obtain the noncommutative (fuzzy) line-bundles by replacing the commuting parameters $\chi_{\alpha}, \chi_{\alpha}^{*}, \alpha=1,2$, by the noncommutative ones, expressing them in terms of annihilation and creation operators as

$$
\hat{\chi}_{\alpha}=A_{\alpha} R^{-1 / 2}, \hat{\chi}_{\alpha}^{*}=R^{-1 / 2} A_{\alpha}^{*},
$$

where

$$
R=A_{\alpha}^{*} A_{\alpha}
$$

so that the condition $\chi_{\alpha}^{*} \chi_{\alpha}=1$ is satisfied (without lack of generality, we choose the unit radius $r=1$ of the sphere). The operators $\chi_{\alpha}$ are well defined on all vectors except vacuum; we complete the definition by postulating that they annihilate the vacuum. The operators $A_{\alpha}$ and $A_{\alpha}^{*}$ (* denotes Hermitian conjugation) act in the Fock space $\mathcal{F}$ spanned by the orthonormal vectors

$$
\left|n_{1}, n_{2}\right\rangle=\frac{1}{\sqrt{n_{1} ! n_{2} !}}\left(A_{1}^{*}\right)^{n_{1}}\left(A_{2}^{*}\right)^{n_{2}}|0\rangle
$$

where $|0\rangle$ is the vacuum defined by $A_{1}|0\rangle=A_{2}|0\rangle=0$. They satisfy in $\mathcal{F}$ the commutation relations

$$
\left[A_{\alpha}, A_{\beta}\right]=\left[A_{\alpha}^{*}, A_{\beta}^{*}\right]=0,\left[A_{\alpha}, A_{\beta}^{*}\right]=\delta_{\alpha \beta} .
$$

The operators $R$ and

$$
R_{j}=\frac{1}{2} A_{\alpha}^{*} \sigma_{\alpha \beta}^{j} A_{\beta}
$$

\footnotetext{
${ }^{4}$ In other words: the section of the line-bundle with the winding number $\kappa$.
} 
satisfy in $\mathcal{F}$ the $u(2)$ algebra commutation relations

$$
\left[R_{i}, R_{j}\right]=i \varepsilon_{i j k} R_{k},\left[R_{i}, R\right]=0 .
$$

Equation (35) is the Schwinger-Jordan realization of the $s u(2)$ algebra. On the other hand, it is just the noncommutative (quantum) version of the Hopf fibration (18).

As $\hat{\mathcal{H}}_{k}, k \in \frac{1}{2} \mathbf{Z}$, we denote the linear space spanned by the normal products

$$
\hat{\chi}_{1}^{* m_{1}} \hat{\chi}_{2}^{* m_{2}} \hat{\chi}_{1}^{n_{1}} \hat{\chi}_{2}^{n_{2}}
$$

with $k=\frac{1}{2}\left(m_{1}+m_{2}-n_{1}-n_{2}\right)$ fixed. Obviously, $\hat{\mathcal{A}}=\hat{\mathcal{H}}_{0}$ is the noncommutative algebra generated by $R, R_{j}, j=1,2,3$ with relations (36). The spaces $\hat{\mathcal{H}}_{k}$ are $\hat{\mathcal{A}}$-bimodules. The operators $J_{j}$ act in $\hat{\mathcal{H}}_{k}$ as follows

$$
J_{j} f=\left[R_{j}, f\right],
$$

and they satisfy in $\hat{\mathcal{H}}_{k}$ the $s u(2)$ algebra commutations relations.

For the following discussion, it is useful to consider $(N+1)$-dimensional subspaces

$$
\mathcal{F}_{N}=\left\{\left|n_{1}, n_{2}\right\rangle, n_{1}+n_{2}=N\right\}, N=0,1,2, \ldots,
$$

of the Fock space $\mathcal{F}$. The operator $R$ takes in $\mathcal{F}_{N}$ the constant value $R=N$. The subspace $\mathcal{F}_{N}$ is the representation space of the unitary irreducible spin $\frac{N}{2}$-representation of the $s u(2)$ algebra in which the Casimir operator

$$
C=R_{3}^{2}+\frac{1}{2}\left(R_{+} R_{-}+R_{-} R_{+}\right), R_{ \pm}=R_{1} \pm i R_{2}
$$

takes the value

$$
C=\frac{N}{2}\left(\frac{N}{2}+1\right) .
$$

As $\hat{\mathcal{H}}_{M N}$ we denote the space of linear mappings from $\mathcal{F}_{N}$ to $\mathcal{F}_{M}$ spanned by the monomials (37) with $m_{1}+m_{2} \leq M, n_{1}+n_{2} \leq N, m_{1}+m_{2}-n_{1}-n_{2}=$ $M-N$. Obviously,

$$
\hat{\mathcal{H}}_{M N}^{*}=\hat{\mathcal{H}}_{N M}, \hat{\mathcal{H}}_{L M} \hat{\mathcal{H}}_{M N} \subset \hat{\mathcal{H}}_{L N} .
$$

Any operator $\Phi \in \hat{\mathcal{H}}_{M N}$ maps $\mathcal{F}_{N}$ to $\mathcal{F}_{M}$, and can be represented by an $(M+1) \times(N+1)$ matrix. There is an antilinear isomorphism between $\hat{\mathcal{H}}_{M N}$ 
and $\hat{\mathcal{H}}_{N M}$ represented as the (matrix) Hermitian conjugation. In $\hat{\mathcal{H}}_{M N}$ we introduce the scalar product

$$
\begin{gathered}
\left(\Phi_{1}, \Phi_{2}\right)_{M N}=\frac{1}{J+1} \operatorname{Tr}_{N}\left(\Phi_{1}^{*} \Phi_{2}\right) \\
=\frac{1}{J+1} \operatorname{Tr}_{M}\left(\Phi_{2} \Phi_{1}^{*}\right)=\left(\Phi_{2}^{*}, \Phi_{1}^{*}\right)_{N M},
\end{gathered}
$$

where $J=\frac{1}{2}(M+N)$ and $\operatorname{Tr}_{N^{\prime}}$ denotes the trace in the space $\hat{\mathcal{H}}_{N^{\prime} N^{\prime}}$.

In particular, $\hat{\mathcal{A}}_{N}=\hat{\mathcal{H}}_{N N}$ is an $(N+1)^{2}$-dimensional algebra generated by $R^{(N)}, R_{j}^{(N)}, j=1,2,3$, where $R^{(N)}, R_{j}^{(N)}$ denote the restriction of $R$ and $R_{j}$ in $\mathcal{F}_{N}$. This restriction generates the algebra homomorphism $\hat{\mathcal{A}} \rightarrow \hat{\mathcal{A}}_{N}$. We point out that in $\hat{\mathcal{A}}_{N}$ there is an additional relation

$$
R^{(N)}-N=0
$$

which expresses the fact that $\mathcal{F}_{N}$ is the space of an irreducible representation of $s u(2)$. To any operator $\Phi \in \mathcal{A}_{N}$ we assign the integral

$$
I_{N}[\Phi]=\frac{1}{N+1} \operatorname{Tr}_{N}(\Phi)
$$

In [6], we proved that for $N \rightarrow \infty$ the algebras $\hat{\mathcal{A}}_{N}$ approach the standard commutative algebra of functions $\mathcal{A}_{\infty}$ and $I_{N}[\Phi] \rightarrow I_{\infty}[\Phi]$. Obviously, $\hat{\mathcal{H}}_{M N}$ is a left $\hat{\mathcal{A}}_{M}$-module and a right $\hat{\mathcal{A}}_{N}$-module.

The generators of $s u(2)$ rotations $J_{j}$ in $\hat{\mathcal{H}}_{M N}$ are given by

$$
J_{j} \Phi=R_{j}^{(M)} \Phi-\Phi R_{j}^{(N)} .
$$

This $s u(2)$ algebra representation is reducible and is equivalent to the direct product of two irreducible $s u(2)$ representations:

$$
\frac{M}{2} \otimes \frac{N}{2}=|k| \oplus(|k|+1) \ldots \oplus J
$$

where $k=\frac{1}{2}(M-N)$ and $J=\frac{1}{2}(M+N)$. This means that any operator $\Phi \in$ $\hat{\mathcal{H}}_{M N}$ can be expanded into operators $\Phi_{J k m}^{j}$, belonging to the representations indicated in (45):

$$
J_{i}^{2} \Phi_{J k m}^{j}=j(j+1) \Phi_{J k m}^{j}, j=|k|,|k|+1, \ldots, J,
$$




$$
J_{3} \Phi_{J k m}^{j}=m \Phi_{J k m}^{j},|m| \leq j .
$$

Putting $J_{ \pm}=J_{1} \pm i J_{2}$ we obtain the highest-weight functions

$$
\Phi_{J k j}^{j}=N_{J k j} \sqrt{\frac{(2 j+1) !}{(j+k) !(j-k) !}} \hat{\chi}_{2}^{* j+k} \hat{\chi}_{1}^{j-k}
$$

satisfying $J_{+} \Phi_{J k j}^{j}=0$. Here the normalization constant $N_{J k j}$ with respect to the scalar product (41) is given by the equation

$$
N_{J k j}=\sqrt{\frac{(J+1)(J+k) !(J-k) !}{(J+j+1) !(J-j) !}}
$$

(we used eqs. (26) on p. 608 and (43) on p. 618 of Ref. [14]). The other normalized functions $\Phi_{J k m}^{j}, m=0, \pm 1, \ldots, \pm j$, in the irreducible representation containing $\Phi_{J k j}^{j}$ are given by

$$
\Phi_{J k m}^{j}=\sqrt{\frac{(j+m) !}{(j-m) !(2 j) !}} J_{-}^{j-m} \Phi_{J k j}^{j} .
$$

Now we are ready to discuss the commutative limit $J \rightarrow \infty, k$ fixed. Not only in this limit do $\chi *$ and $\chi$ commute among themselves, also the normalization factor $N_{J k j}$ approaches 1 and expression (46) becomes the standard Wigner $D$-function $D_{k j}^{j}$, expressed in terms of $\chi_{\alpha}, \chi_{\alpha}^{*}$ instead of Euler angles. Since $\left[J_{i}, R\right]=0$, the same remains true for the functions $\Phi_{J k m}^{j}$ given in (48). The normalization coefficient $N_{J k j}$ is also a cut-off factor, as can be seen from (47), because $N_{J k j}=0$ for $j>J$. If we vary $k$ while keeping $J$ fixed, then $\chi *$ and $\chi$ will cease to commute for $J-|k| \rightarrow 0$, even though $J$ can be very large. This is in accordance with the general principle (cf. 6]) that approaching the maximal spin $J$ of the truncation the multiplication becomes noncommutative.

In the noncommutative case we identify a section $\Phi$ of a complex line bundle with fixed winding number with an element of $\hat{\mathcal{H}}_{M N}$. The corresponding field theory action we take in the form

$$
S_{M N}\left[\Phi, \Phi^{*}\right]=\frac{1}{J+1} \operatorname{Tr}_{N}\left[\frac{1}{2} \Phi^{*}\left(K_{+} K_{-}+K_{-} K_{+}\right) \Phi+V\left(\Phi^{*} \Phi\right)\right]
$$


where in the noncommutative case the operators $K_{ \pm}$are defined by

$$
K_{+} \Phi=i \varepsilon_{\alpha \beta} A_{\beta}^{*}\left[\Phi, A_{\alpha}^{*}\right], K_{-} \Phi=i \varepsilon_{\alpha \beta}\left[A_{\alpha}, \Phi\right] A_{\beta} .
$$

Note that the 'topological charge' operator $K_{0}$ defined as

$$
K_{0} \Phi=\frac{1}{2}[R, \Phi]
$$

takes in $\hat{\mathcal{H}}_{M N}$ the constant value $k=\frac{1}{2}(M-N)$. The order of operators in (50) is essential because it guarantees that the operators $K_{ \pm}$act on monomials exactly in the same way as in the commutative case.

Note: We would like to stress that for the description of topologically nontrivial field configurations (with $\kappa \neq 0$ ), two algebras $\hat{\mathcal{A}}_{M}$ and $\hat{\mathcal{A}}_{N}$ (with $M-N=\kappa \neq 0$ ) are needed. This is the reason why the discussion within only one matrix algebra $(M=N)$ corresponds to the topologically trivial situation (see e.g. 因, 局, 6, 11]).

If the winding number of the field $\Phi$ is not fixed, we work with fields from the space

$$
\hat{\mathcal{H}}_{(J)}=\bigoplus_{M+N=2 J} \hat{\mathcal{H}}_{M N},
$$

and the corresponding action we take as

$$
S_{(J)}\left[\Phi, \Phi^{*}\right]=\sum_{M+N=2 J} S_{M N}\left[\Phi, \Phi^{*}\right] .
$$

The action (53) has the following basic properties:

1) it has the full $s u(2)$ symmetry corresponding to the rotations of $S^{2}$;

2 ) it describes a model with a finite number of modes since, in fact, it corresponds to a particular matrix model; and

3 ) it approaches in the limit $J \rightarrow \infty$ the commutative action (for any given polynomial field $\Phi$ ).

In general, the complex scalar field from $\hat{\mathcal{H}}_{(J)}$ can be expanded as

$$
\Phi=\sum_{j=0}^{J} \sum_{k, m=-j}^{+j} a_{k m}^{j} \Phi_{J k m}^{j} .
$$

The quantum field mean value of a functional $F\left[\Phi, \Phi^{*}\right]$ is defined as

$$
\left\langle F\left[\Phi, \Phi^{*}\right]\right\rangle=\frac{\int D \Phi D \Phi^{*} e^{-S_{(J)}\left[\Phi, \Phi^{*}\right]} F\left[\Phi, \Phi^{*}\right]}{\int D \Phi D \Phi^{*} e^{-S_{(J)}\left[\Phi, \Phi^{*}\right]}},
$$


where $D \Phi D \Phi^{*}=\prod_{j k m} d a_{k m}^{j} d a_{k m}^{* j}$ is a finite product of the standard measures in the complex plane. The quantum mean values are well defined for any polynomial functional $F\left[\Phi, \Phi^{*}\right]$.

Under rotations specified by the Euler angles $\alpha, \beta, \gamma$ the coefficients of the field expansion transform as

$$
\begin{aligned}
& a_{k m}^{j} \rightarrow a_{k m^{\prime}}^{\prime j}=\sum_{m} D_{m^{\prime} m}^{j}(\alpha, \beta, \gamma) a_{k m}^{j}, \\
& a_{k m}^{* j} \rightarrow a_{k m^{\prime}}^{\prime * j}=\sum_{m} D_{m^{\prime} m}^{* j}(\alpha, \beta, \gamma) a_{k m}^{* j} .
\end{aligned}
$$

These are the unitary transformations not changing the measure $D \Phi D \Phi^{*}$. This completes the proof of rotational invariance of the model at the quantum level.

\section{Spinor fields}

For construction of the topologically nontrivial spinor fields we use the superspace approach developed in [6]. First we perform the $\mathcal{N}=1$ superextension of the Hopf fibration described in the previous section. We obtain it from the mapping $\mathbf{C}^{2,1} \rightarrow \mathbf{R}^{3,2}$ given by

$$
\xi=\left(\begin{array}{c}
\chi_{1} \\
\chi_{2} \\
a
\end{array}\right) \rightarrow\left(x_{i}, \theta_{\mu}\right), i=1,2,3, \mu=+,-
$$

where

$$
x_{i}=\xi^{+} \Sigma_{i} \xi, \theta_{\mu}=\xi^{+} F_{\mu} \xi .
$$

Here $\xi^{+}=\left(\chi_{1}^{*}, \chi_{2}^{*}, a^{*}\right)$, and $\Sigma_{ \pm}=\Sigma_{1} \pm i \Sigma_{2}, \Sigma_{3}$ and $F_{ \pm}$are $3 \times 3$ matrices given by

$$
\begin{gathered}
\Sigma_{+}=\left(\begin{array}{lll}
0 & 1 & 0 \\
0 & 0 & 0 \\
0 & 0 & 0
\end{array}\right), \Sigma_{-}=\left(\begin{array}{lll}
0 & 0 & 0 \\
1 & 0 & 0 \\
0 & 0 & 0
\end{array}\right), \Sigma_{3}=\frac{1}{2}\left(\begin{array}{ccc}
1 & 0 & 0 \\
0 & -1 & 0 \\
0 & 0 & 0
\end{array}\right), \\
F_{+}=\frac{1}{\sqrt{2}}\left(\begin{array}{ccc}
0 & 0 & -1 \\
0 & 0 & 0 \\
0 & -1 & 0
\end{array}\right), F_{-}=\frac{1}{\sqrt{2}}\left(\begin{array}{ccc}
0 & 0 & 0 \\
0 & 0 & -1 \\
1 & 0 & 0
\end{array}\right)
\end{gathered}
$$


The quantities $\chi_{\alpha}, \chi_{\alpha}^{*}(\alpha=1,2)$ and $a, a^{*}$ are respectively even and odd coordinates of the superspace $C^{2,1}$; ${ }^{*}$ denotes the graded involution [15, 6] characterized by the properties

$$
(a b)^{*}=(-1)^{\operatorname{deg} a \operatorname{deg} b} b^{*} a^{*}, \quad a^{* *}=(-1)^{\operatorname{deg} a} a .
$$

Note that a restriction $\chi_{1}^{*} \chi_{1}+\chi_{2}^{*} \chi_{2}+a^{*} a=2 \rho$ implies $x_{i}^{2}+\theta_{+} \theta_{-}=\rho^{2}$. Thus we obtain the Hopf superfibration $s S^{3} \rightarrow s S^{2}$ of the 3 -supersphere in $C^{2,1}$ with the fibration basis being the two-supersphere in $R^{3,2}$.

It is worth noting that $\Sigma_{i}$ and $F_{\mu}$ are respectively even and odd generators of the $\operatorname{osp}(2,1)$ superalgebra:

$$
\begin{gathered}
{\left[\Sigma_{3}, \Sigma_{ \pm}\right]= \pm \Sigma_{ \pm},\left[\Sigma_{+}, \Sigma_{-}\right]=2 \Sigma_{3}} \\
{\left[\Sigma_{3}, F_{ \pm}\right]= \pm \frac{1}{2} F_{ \pm},\left[\Sigma_{ \pm}, F_{ \pm}\right]=0,\left[\Sigma_{ \pm}, F_{\mp}\right]=F_{ \pm}} \\
{\left[F_{ \pm}, F_{ \pm}\right]= \pm \Sigma_{ \pm},\left[F_{+}, F_{-}\right]=-\Sigma_{3} .}
\end{gathered}
$$

Here and in what follows, the symbol $[A, B]$ denotes a supercommutator, i.e. the commutator $A B-B A$ if either $A$ or $B$ is even, and the anticommutator $A B+B A$ if both $A$ and $B$ are odd.

A superfunction $\Phi=\Phi\left(\xi, \xi^{*}\right)$ on $s S^{3}$ is represented as a linear combination of monomials

$$
\chi_{1}^{* m_{1}} \chi_{2}^{* m_{2}} \chi_{1}^{n_{1}} \chi_{2}^{n_{2}} a^{* \mu} a^{\nu}
$$

where $m_{\alpha}, n_{\alpha}$ are non-negative integers and $\mu, \nu=0,1$ f The representatives, which are identical on the surface $\chi_{1}^{*} \chi_{1}+\chi_{2}^{*} \chi_{2}+a^{*} a=r$, correspond to the same superfunction $\Phi$ on $s S^{3}$. To any such monomial we assign the topological charge $2 k=m_{1}+m_{2}+\mu-n_{1}-n_{2}-\nu$. As $s \mathcal{H}_{k}, k \in \frac{1}{2} \mathbf{Z}$, we denote the linear space spanned by the monomials with fixed $k$. Any superfunction $\Phi \in s \mathcal{H}_{k}$ can be expanded as

$$
\Phi=\Phi_{0}\left(\chi, \chi^{*}\right)+f\left(\chi, \chi^{*}\right) a+g\left(\chi, \chi^{*}\right) a^{*}+F\left(\chi, \chi^{*}\right) a^{*} a
$$

where $\Phi_{0}, F \in \mathcal{H}_{k}$ are even and $f \in \mathcal{H}_{k+\frac{1}{2}}, g \in \mathcal{H}_{k-\frac{1}{2}}$ are odd. The space $s \mathcal{H}_{0}$ is a superalgebra $s \mathcal{A}$ with respect to the supercommutative 'point-wise' product of parameters $\xi, \xi^{*}$. The spaces $s \mathcal{H}_{k}$ are $s \mathcal{A}$-bimodules.

\footnotetext{
${ }^{5}$ If the monomial in (59) is odd, then it appears with an odd coefficient in the decomposition of the superfunction $\Phi$ in the linear combination of the monomials (59). Thus the superfunction $\Phi$ itself is an even element of the Grassmann algebra.
} 
The differential operators generating the $\operatorname{osp}(2,1)$ algebra acting on $s \mathcal{H}_{k}$ are given by

$$
\begin{aligned}
J_{i} & =\frac{i}{2}\left[\xi_{a}^{*} \Sigma_{a b}^{i} \partial_{\xi_{b}^{*}}-\xi_{a} \Sigma_{a b}^{* i} \partial_{\xi_{b}}\right], \\
v_{\mu} & =\frac{i}{2}\left[\xi_{a}^{*} F_{a b}^{\mu} \partial_{\xi_{b}^{*}}+\xi_{a} F_{a b}^{* \mu} \partial_{\xi_{b}}\right] .
\end{aligned}
$$

The function $C(x, \theta)=x_{i}^{2}+\frac{1}{2}\left(\theta_{+} \theta_{-}-\theta_{-} \theta_{+}\right)$is an invariant superfunction from $s \mathcal{H}_{0}$ :

$$
J_{i} C(x, \theta)=0, v_{\mu} C(x, \theta)=0 .
$$

The condition $C(x, \theta)=\rho^{2}$ defines a supersphere in $\mathbf{R}^{3,2}$.

In $s \mathcal{H}_{0}$ we can introduce a standard Berezin integral over the supersphere [6] as

$$
s I_{\infty}[\Phi]=\frac{\rho}{\pi} \int d^{3} x d \theta_{+} d \theta_{-} \delta\left(x_{i}^{2}+\theta_{+} \theta_{-}-\rho^{2}\right) \Phi(x, \theta),
$$

where the super $\delta$-function should be understood as

$$
\delta\left(x_{i}^{2}+\theta_{+} \theta_{-}-\rho^{2}\right)=\delta\left(x_{i}^{2}-\rho^{2}\right)+\theta_{+} \theta_{-} \delta^{\prime}\left(x_{i}^{2}-\rho^{2}\right),
$$

(this formula is obtained by a Taylor expansion of the super $\delta$-function). Expressing $\theta_{\mu}$ in terms of $a, a^{*}$ we obtain

$$
s I_{\infty}[\Phi]=\frac{1}{\pi} \int d^{3} x d a^{*} d a\left[\delta\left(x_{i}^{2}-\rho^{2}\right)+\rho a^{*} a \delta^{\prime}\left(x_{i}^{2}-\rho^{2}\right)\right] \Phi(x, a),
$$

where one can now expand $\Phi$ directly as in (60). Much as before, we can introduce the inner product in $s \mathcal{H}_{k}$

$$
\left(\Phi_{1}, \Phi_{2}\right)_{k}=s I_{\infty}\left[\Phi_{1}^{*} \Phi_{2}\right]
$$

where (cf. (60))

$$
\Phi^{*}=\Phi_{0}^{*}\left(\chi, \chi^{*}\right)-g^{*} a+f^{*} a^{*}+F^{*} a^{*} a .
$$

The spinor field $\Psi$ we identify with the odd part of the superfield $\Phi$ :

$$
\Psi=f\left(\chi, \chi^{*}\right) a+g\left(\chi, \chi^{*}\right) a^{*}
$$

where $f$ and $g$ are also odd (cf. footnote 5). As $\mathcal{S}_{k}, k \in \frac{1}{2} \mathbf{Z}$ we denote the set of spinor fields from $s \mathcal{H}_{k}$, i.e. with $f \in \mathcal{H}_{k+\frac{1}{2}}$ and $g \in \mathcal{H}_{k-\frac{1}{2}} ; \mathcal{S}_{k}$ 
are $\mathcal{A}$-bimodules (but not $s \mathcal{A}$-(bi)modules). The formula (66) induces the decomposition of the spinor space:

$$
\mathcal{S}_{k}=\mathcal{S}_{k}^{(+)} \oplus \mathcal{S}_{k}^{(-)}
$$

where $\mathcal{S}_{k}^{(+)}$corresponds to the first term in (66), and $\mathcal{S}_{k}^{(-)}$to the second one. The chirality grading operator $\Gamma$ we define as the operator taking in $\mathcal{S}_{k}^{(\mu)}$ the value $\mu= \pm 1$ :

$$
\Gamma \Psi=f\left(\chi, \chi^{*}\right) a-g\left(\chi, \chi^{*}\right) a^{*} .
$$

The operator $\Gamma$ can be realized as the differential operator

$$
\Gamma \Psi=\left(a \partial_{a}-a^{*} \partial_{a *}\right) \Psi
$$

We define the free Dirac operator $D$ as the following mapping from $\mathcal{S}_{k}$ to $\mathcal{S}_{k}$ :

$$
D \Psi=\left(K_{+} g\right) a+\left(K_{-} f\right) a^{*} .
$$

It can be expressed as the second-order (in even and odd parameters) differential operator

$$
D \Psi=\left(a \partial_{a^{*}} K_{-}+a^{*} \partial_{a} K_{+}\right) \Psi .
$$

The Dirac operator anticommutes with the chirality operator:

$$
D \Gamma+\Gamma D=0
$$

The action for spinor field we define in terms of the Berezin integral

$$
S\left[\Psi, \Psi^{*}\right]=s I_{\infty}\left[\Psi^{*} D \Psi+W\left(\Psi^{*}, \Psi\right)\right]
$$

where $W\left(\Psi^{*}, \Psi\right)$ is a suitable potential describing the self-interaction of the spinor field.

Note: We would like to stress that the formalism for the spinor field presented above is equivalent to the usual one described in Section 2, but it is better suited for the non-commutative generalization.

In the noncommutative case, we replace $\chi_{\alpha}, \chi_{\alpha}^{*}$, and $a, a^{*}$ by bosonic and fermionic annihilation and creation operators

$$
\begin{gathered}
\hat{\chi}_{\alpha}=A_{\alpha} R^{-1 / 2}, \hat{\chi}_{\alpha}^{*}=R^{-1 / 2} A_{\alpha}^{*}, \\
\hat{a}=b R^{-1 / 2}, \hat{a}^{*}=R^{-1 / 2} b^{*},
\end{gathered}
$$


where now

$$
R=A_{\alpha}^{*} A_{\alpha}+b^{*} b,
$$

so that the condition $\chi_{\alpha}^{*} \chi_{\alpha}+a^{*} a=1$ is satisfied (this corresponds to the radius of the two-sphere $\rho=1 / 2$ ). The operators $A_{\alpha}, A_{\alpha}^{*}, b, b^{*}$ act in the Fock space $s \mathcal{F}$ spanned by the orthonormal vectors

$$
\left|n_{1}, n_{2} ; \nu\right\rangle=\frac{1}{\sqrt{n_{1} ! n_{2} !}}\left(A_{1}^{*}\right)^{n_{1}}\left(A_{2}^{*}\right)^{n_{2}}\left(b^{*}\right)^{\nu}|0\rangle,
$$

where $n_{1}, n_{2}$ are non-negative integers, $\nu=0,1$ and $|0\rangle$ is the vacuum defined by $A_{1}|0\rangle=A_{2}|0\rangle=b|0\rangle=0$. The annihilation and creation operators in question satisfy in $s \mathcal{F}$ the supercommutation relations

$$
\begin{gathered}
{\left[A_{\alpha}, A_{\beta}\right]=\left[A_{\alpha}^{*}, A_{\beta}^{*}\right]=0,\left[A_{\alpha}, A_{\beta}^{*}\right]=\delta_{\alpha, \beta} .} \\
{\left[A_{\alpha}, b\right]=\left[A_{\alpha}^{*}, b\right]=\left[A_{\alpha}, b^{*}\right]=\left[A_{\alpha}^{*}, b^{*}\right]=0,} \\
b^{2}=\left(b^{*}\right)^{2}=0,\left[b, b^{*}\right]=1 .
\end{gathered}
$$

The operators

$$
\begin{gathered}
R_{j}=\frac{1}{2} A_{\alpha}^{*} \sigma_{\alpha \beta}^{j} A_{\beta}, \\
V_{+}=\frac{1}{\sqrt{2}}\left(-A_{1}^{*} b-b^{*} A_{2}\right), V_{-}=\frac{1}{\sqrt{2}}\left(-A_{2}^{*} b+b^{*} A_{1}\right),
\end{gathered}
$$

then satisfy in the Fock space $s \mathcal{F}$ the $\operatorname{osp}(2,1)$ superalgebra commutation relations (58) (with an obvious change in the notation).

As $s \hat{\mathcal{H}}_{k}$ we denote the linear space of superfields (60) with $\Phi_{0}, F \in \hat{\mathcal{H}}_{k}$, $f \in \hat{\mathcal{H}}_{k+\frac{1}{2}}$ and $g \in \hat{\mathcal{H}}_{k-\frac{1}{2}}$. Obviously, $s \mathcal{A}=s \hat{\mathcal{H}}_{0}$ is a superalgebra, and $s \hat{\mathcal{H}}_{k}$ are $s \mathcal{A}$-bimodules. The subalgebra $\mathcal{A}$ is naturally embedded into $s \mathcal{A}$ (as the set of $\Phi_{0}$ 's in the decomposition (60) for $k=0$ ). The generators $J_{j}$ and $\mathcal{V}_{\mu}$ of $\operatorname{osp}(2,1)$ act in the space $s \hat{\mathcal{H}}_{k}$ by means of the superadjoint action (cf. [6])

$$
J_{j} \Phi=\left[R_{j}, \Phi\right], \mathcal{V}_{\mu} \Phi=\left[V_{\mu}, \Phi\right] .
$$

Obviously, this is a reducible representation of the superalgebra $\operatorname{osp}(2,1)$ in $s \hat{\mathcal{H}}_{k}$.

\footnotetext{
${ }^{6}$ Note that ${ }^{*}$ still denotes the graded involution. This means that $b^{*}$ is the adjoint operator $b^{\dagger}$ of $b$ in the standard fermionic Fock space, but $b^{* *}=-b$.
} 
The spinor field $\Psi$ we identify with the odd part of the superfield $\Phi$ :

$$
\Psi=f\left(\hat{\chi}^{*}, \hat{\chi}\right) \hat{a}+g\left(\hat{\chi}^{*}, \hat{\chi}\right) \hat{a}^{*} .
$$

As $\hat{\mathcal{S}}_{k}, k \in \frac{1}{2} \mathbf{Z}$ we denote the set of spinor fields from $s \hat{\mathcal{H}}_{k}$, i.e. with $f \in \hat{\mathcal{H}}_{k+\frac{1}{2}}$ and $g \in \hat{\mathcal{H}}_{k-\frac{1}{2}}$. $\hat{\mathcal{S}}_{k}$ are $\mathcal{A}$-bimodules (but not $s \mathcal{A}$-(bi)modules).

Formula (79) induces the decomposition of the spinor space:

$$
\hat{\mathcal{S}}_{k}=\hat{\mathcal{S}}_{k}^{(+)} \oplus \hat{\mathcal{S}}_{k}^{(-)},
$$

where $\hat{\mathcal{S}}_{k}^{(+)}$corresponds to the first term in $(79)$, and $\hat{\mathcal{S}}_{k}^{(-)}$to the second one. The grading $\hat{\Gamma}$ we define in the same way as in (68):

$$
\hat{\Gamma} \Psi=f\left(\hat{\chi}^{*}, \hat{\chi}\right) \hat{a}-g\left(\hat{\chi}^{*}, \hat{\chi}\right) \hat{a}^{*} .
$$

It can also be written as

$$
\hat{\Gamma} \Psi=-\left[b^{*} b, \Psi\right] .
$$

Thus the grading is directly related to the fermion number and it takes, in $\hat{\mathcal{S}}_{k}^{(\mu)}$, the value $\mu= \pm 1$.

The free Dirac operator $D$ we define as in (70):

$$
D \Psi=\left(K_{+} g\right) \hat{a}+\left(K_{-} f\right) \hat{a}^{*},
$$

where $K_{ \pm}$were defined in (50). The Dirac operator maps $\hat{\mathcal{S}}_{k}$ into $\hat{\mathcal{S}}_{k}$ and it anticommutes with the chirality grading operator:

$$
D \Gamma+\Gamma D=0 \text {. }
$$

As $s \mathcal{F}_{N}$ we denote the subspace

$$
s \mathcal{F}_{N}=\left\{\left|n_{1}, n_{2} ; \nu\right\rangle \in s \mathcal{F}, n_{1}+n_{2}+\nu=N\right\},
$$

in which the operator $R$ takes the value $R=N$. Obviously, $s \mathcal{F}_{N}$ is a $(2 N+1)$ dimensional superspace

$$
s \mathcal{F}_{N}=s \mathcal{F}_{N}^{(0)} \oplus s \mathcal{F}_{N-1}^{(1)}
$$

where $s \mathcal{F}_{N^{\prime}}^{(\nu)}$ is the subspace with $N^{\prime}$ bosons and $\nu$ fermions, $s \mathcal{F}_{N}^{(0)}$ is the even subspace of $s \mathcal{F}_{N}$, and $s \mathcal{F}_{N-1}^{(1)}$ is the odd one. The space $s \mathcal{F}_{N}$ is the 
representation space of the irreducible representation of the superalgebra $\operatorname{osp}(2,1)$ (generated by $R_{j}, V_{\mu}$ given above) in which the Casimir operator

$$
C=R_{3}^{2}+\frac{1}{2}\left(R_{+} R_{-}+R_{-} R_{+}\right)+\frac{1}{2}\left(V_{+} V_{-}-V_{-} V_{+}\right)
$$

takes the value

$$
C=\frac{1}{4} N(N+1)
$$

As $s \hat{\mathcal{H}}_{M N}$ we denote the space of linear mappings from $s \mathcal{F}_{N}$ to $s \mathcal{F}_{M}$ spanned by the operator monomials

$$
\hat{\chi}_{1}^{* m_{1}} \hat{\chi}_{2}^{* m_{2}} \hat{\chi}_{1}^{n_{1}} \hat{\chi}_{2}^{n_{2}} \hat{a}^{* \mu} \hat{a}^{\nu}
$$

with $m_{1}+m_{2}+\mu \leq M, n_{1}+n_{2}+\nu \leq N$ and $m_{1}+m_{2}+\mu-n_{1}-n_{2}-\nu=M-N$. Any operator $\Phi \in s \hat{\mathcal{H}}_{M N}$ can be represented by a $(2 N+1) \times(2 M+1)$ supermatrix. In $s \hat{\mathcal{H}}_{M N}$ we introduce an inner product

$$
\left(\Phi_{1}, \Phi_{2}\right)_{M N}=s \operatorname{Tr}_{N}\left(\Phi_{1}^{*} \Phi_{2}\right),
$$

where $J=\frac{1}{2}(M+N)$ and $s \operatorname{Tr}_{N}$ denotes the supertrace in the space $s \hat{\mathcal{H}}_{N N}$. As in the purely bosonic case, the action of $s u(2)$ generators $J_{i}$ on $s \hat{\mathcal{H}}_{M N}$ is given by the formula (44) with $R_{i}$ given by (77).

As $\hat{\mathcal{S}}_{M N}$ we denote the space of spinor fields from $s \hat{\mathcal{H}}_{M N}$, i.e.

$$
\Psi=f\left(\hat{\chi}^{*}, \hat{\chi}\right) \hat{a}+g\left(\hat{\chi}^{*}, \hat{\chi}\right) \hat{a}^{*},
$$

where $f \in \hat{\mathcal{H}}_{M, N-1}$ and $g \in \hat{\mathcal{H}}_{M-1, N}$. This gives the decomposition

$$
\hat{\mathcal{S}}_{M N}=\hat{\mathcal{S}}_{M N}^{(+)} \oplus \hat{\mathcal{S}}_{M N}^{(-)},
$$

where $\hat{\mathcal{S}}_{M N}^{(\mu)}$ contains spinor fields with the chirality $\mu= \pm 1$. The operators $K_{ \pm}$, entering the Dirac operator $D(83)$, act in $\hat{\mathcal{S}}_{M N}$; moreover, $K_{0}$ takes in $\hat{\mathcal{S}}_{M N}^{(\mu)}$ the constant value $\frac{1}{2}(M-N+\mu)$. The spinor field operators from $\hat{\mathcal{S}}_{M N}^{(\mu)}$ are odd mappings $s \mathcal{F}_{N} \rightarrow s \mathcal{F}_{M}$, namely:

$$
f \hat{a}: s \mathcal{F}_{N}^{(1)} \rightarrow s \mathcal{F}_{M}^{(0)}, \quad g \hat{a}^{*}: s \mathcal{F}_{N}^{(0)} \rightarrow s \mathcal{F}_{M}^{(1)} .
$$

According to (45), $f$ and $g$ can be expanded into operator functions belonging to the representations:

$$
\frac{M}{2} \otimes \frac{N-1}{2}=\left|k+\frac{1}{2}\right| \oplus \ldots \oplus\left(J-\frac{1}{2}\right), \text { for } f,
$$




$$
\frac{M-1}{2} \otimes \frac{N}{2}=\left|k-\frac{1}{2}\right| \oplus \ldots \oplus\left(J-\frac{1}{2}\right), \text { for } g,
$$

where $k=\frac{1}{2}(M-N)$ and $J=\frac{1}{2}(M+N)$. This means that $f$ can be expanded into the functions $\Phi_{J-\frac{1}{2}, k+\frac{1}{2}, m}^{j}$ with $j=\left|k+\frac{1}{2}\right|, \ldots, J-\frac{1}{2},|m| \leq j$, and, in the same way, $g$ into the functions $\Phi_{J-\frac{1}{2}, k-\frac{1}{2}, m}^{j}$ with $j=\left|k-\frac{1}{2}\right|, \ldots, J-\frac{1}{2}$, $|m| \leq j$ (cf. (48)). The admissible values of $j$ are:

$$
j=|k|-\frac{1}{2},|k|+\frac{1}{2}, \ldots, J-\frac{1}{2} .
$$

They can be seen from the representation content in Eqs. (91):

(i) The first value $j=|k|-\frac{1}{2}$ is admissible only for $k \neq 0$, and for $k>0$ it is present in $g$ (chirality -1 ), whereas for $k<0$ it is present in $f$ (chirality +1 ). It corresponds to the $|M-N|$ (normalized) zero modes of the Dirac operator given by

$$
\begin{gathered}
\Psi_{m_{1} m_{2}}^{0+}=\frac{\sqrt{\left(m_{1}+m_{2}+1\right) !}}{\sqrt{m_{1} ! m_{2} !}} \hat{\chi}_{1}^{* m_{1}} \hat{\chi}_{2}^{* m_{2}} \hat{a}^{*}, \text { for } k=\frac{1}{2}\left(m_{1}+m_{2}+1\right)>0 \\
\left.\Psi_{n_{1} n_{2}}^{0}=\frac{\sqrt{\left(n_{1}+n_{2}+1\right) !}}{\sqrt{n_{1} ! n_{2} !}} \hat{\chi}_{1}^{n_{1}} \hat{\chi}_{2}^{n_{2}} \hat{a}, \text { for } k=-\frac{1}{2}\left(n_{1}+n_{2}+1\right)<0, \quad \text {, } 92\right)
\end{gathered}
$$

Note that the normalization does not depend on the cut-off spin $J$; therefore the correct commutative limit is obvious.

(ii) The remaining eigenvalues $j=|k|+\frac{1}{2}, \ldots, J-\frac{1}{2}$, correspond to nonzero modes of the Dirac operator. Consider (normalized) functions $\Phi_{J k m}^{j \pm}$ with a given chirality $\mu= \pm 1$ given by

$$
\Phi_{J k m}^{j-}=\Phi_{J-\frac{1}{2}, k-\frac{1}{2}, m}^{j} \hat{a}^{*}, \Phi_{J k m}^{j+}=\Phi_{J-\frac{1}{2}, k+\frac{1}{2}, m}^{j} \hat{a}
$$

where $\Phi_{J k^{\prime} m}^{j}$ were defined in (46) and (48). Using the definition of the Dirac operator (83) and the formula

$$
K_{ \pm} \Phi_{J l m}^{j}=\sqrt{(j \pm l+1)(j \mp l)} \Phi_{J, l \pm 1, m}^{j},
$$

\footnotetext{
${ }^{7}$ Formula (94) follows from the properties of the Wigner functions (cf. the discussion after (48)) because $K_{ \pm}$act on the second subscript of $\Phi_{J l m}^{j}$ in exactly the same way as $J_{ \pm}$act on the third subscript.
} 
we obtain the equation

$$
D \Phi_{J k m}^{j \pm}=E_{k j} \Phi_{J k m}^{j \mp}
$$

where

$$
E_{k j}=\sqrt{\left(j+\frac{1}{2}\right)^{2}-k^{2}} .
$$

Thus the functions

$$
\Psi_{J k m}^{j \pm}=\frac{1}{\sqrt{2}} \Phi_{J k m}^{j+} \pm \frac{1}{\sqrt{2}} \Phi_{J k m}^{j-}
$$

are (normalized) eigenfuctions of the Dirac operator with the eigenvalues $\pm E_{k j}$.

Note: We would like to stress that in the standard commutative case exactly the same formula is obtained for the spectrum, except for the fact that the admissible values of $j$ are not truncated. As the number of degrees of freedom is finite this will lead to a non-perturbative UV-regularization (for a real scalar field this was demonstrated in [5]).

The action for the self-interacting (Dirac) spinor field $\Psi \in \hat{\mathcal{S}}_{M N}$ with a fixed winding number we define as follows

$$
S_{M N}\left[\Psi^{*}, \Psi\right]=s \operatorname{Tr}_{N}\left[\Psi^{*} D \Psi+W\left(\Psi^{*}, \Psi\right)\right]
$$

where $\Psi=f a+g a^{*}, \Psi^{*}=a g^{*}-a^{*} f^{*}$, and $W(.,$.$) is an interaction term.$ We do not wish to fix the winding number of the field $\Psi$; instead we take $\Psi$ from the space

$$
\hat{\mathcal{S}}_{(J)}=\bigoplus_{M+N=2 J} \hat{\mathcal{S}}_{M N},
$$

and we define the corresponding action as

$$
S_{(J)}\left[\Psi^{*}, \Psi\right]=\sum_{M+N=2 J} S_{M N}\left[\Psi^{*}, \Psi\right]
$$

This action has the following basic properties:

1) It is invariant with respect to the space isometries, i.e. the rotations of the sphere, and the chiral transformations

$$
\Psi \rightarrow e^{i \alpha \Gamma} \Psi, \Psi^{*} \rightarrow \Psi^{*} e^{i \alpha \Gamma}, \alpha \in \mathbf{R}
$$


provided that the interaction term is rotationally and chirally invariant.

2) It describes the system with a finite number of degrees of freedom.

3) It approaches for $J \rightarrow \infty$ the commutative action.

Note: The rotational invariance of the action $S_{M N}$ follows from the rotational invariance of the truncated Dirac operator. The chiral invariance of the action is obvious, because the Dirac operator $D$ anticommutes with the grading $\Gamma$ (cf. (84)).

The spinor field $\Psi \in \hat{\mathcal{S}}_{M N}$ can be expanded as

$$
\Psi=\sum_{m_{1} m_{2}} a_{m_{1} m_{2}}^{0,|k|-\frac{1}{2}} \Psi_{m_{1} m_{2}}^{0}+\sum_{j=|k|+\frac{1}{2}}^{J-\frac{1}{2}} \sum_{m=-j}^{+j}\left(a_{k m}^{j+} \Phi_{J k m}^{j+}+a_{k m}^{j-} \Phi_{J k m}^{j-}\right),
$$

where the first sum corresponds to the zero modes (92) and the remaining two sums are related to the non-zero modes (96). All expansion coefficients $a_{. .}$in (102) are supposed to be independent anticommuting Grassmannian variables. In the same way, the field $\Psi^{*} \in \hat{\mathcal{S}}_{N M}$ is supposed to have an expansion with independent Grassmann coefficients $a_{. . .}^{* .}$.

The quantum field mean value of a functional $F\left[\ddot{\Psi}, \Psi^{*}\right]$ is given as

$$
\left\langle F\left[\Psi, \Psi^{*}\right]\right\rangle_{M N}=\frac{\int[D \Psi]_{M N}\left[D \Psi^{*}\right]_{N M} e^{-S_{M N}\left[\Psi, \Psi^{*}\right]} F\left[\Psi, \Psi^{*}\right]}{\int[D \Psi]_{M N}\left[D \Psi^{*}\right]_{N M} e^{-S_{M N}\left[\Psi, \Psi^{*}\right]}},
$$

where $\int[D \Psi]_{M N}\left[D \Psi^{*}\right]_{N M} \ldots$ denotes the finite-dimensional Berezin integral over all admissible coefficients $a_{. .}^{. .}$and $a_{. . .}^{* .}$ with fixed $k=\frac{1}{2}(M-N)$ and $J=\frac{1}{2}(M+N)$.

Taking into account that $\Phi_{J k m}^{j+}$ and the zero modes for $k>0$ correspond to $g$, and similarly $\Phi_{J k m}^{j-}$ and the zero modes for $k<0$ correspond to $f$, the chiral transformations (101) can be written as

$$
a_{m_{1} m_{2}}^{0,|k|-\frac{1}{2}} \rightarrow e^{-i \varepsilon(k) \alpha} a_{m_{1} m_{2}}^{0,|k|-\frac{1}{2}}, a_{k m}^{j \pm} \rightarrow e^{\mp i \alpha} a_{k m}^{j \pm},
$$

where $\varepsilon(k)$ is the sign function. We see that the phase contributions to the measure $[D \Psi]_{M N}$ from the non-zero modes cancel. As expected, the only contribution comes from $|\kappa|$ zero modes $(\kappa=M-N)$ :

$$
[D \Psi]_{M N} \rightarrow e^{-i \kappa \alpha}[D \Psi]_{M N} .
$$

Analogous rules are valid for expansion coefficients of the field $\Psi^{*}$. The phase factors from non-zero modes cancel, and there is just the same phase 
contribution from zero modes (since the conjugation and the change $M-N \rightarrow$ $N-M$ compensate each other in the phase factors):

$$
\left[D \Psi^{*}\right]_{N M} \rightarrow e^{-i \kappa \alpha}\left[D \Psi^{*}\right]_{N M} .
$$

Thus, the total change of the measure $[D \Psi]_{M N}\left[D \Psi^{*}\right]_{N M}$ is

$$
[D \Psi]_{M N}\left[D \Psi^{*}\right]_{N M} \rightarrow e^{-2 i \kappa \alpha}[D \Psi]_{M N}\left[D \Psi^{*}\right]_{N M} .
$$

We see that assuming fixed $k \neq 0$, the chiral symmetry is violated on quantum level. However, simultaneously taking into account fields with given $k$ and $-k$, as e.g. in $\hat{\mathcal{S}}_{(J)}$, the chiral symmetry is restored. Thus, the measure $D \Psi D \Psi^{*}=\prod_{M N}[D \Psi]_{M N}\left[D \Psi^{*}\right]_{N M}$ entering the quantum mean value over $\hat{\mathcal{S}}_{(J)}$,

$$
\left\langle F\left[\Psi, \Psi^{*}\right]\right\rangle=\frac{\int D \Psi D \Psi^{*} e^{-S_{(J)}\left[\Psi, \Psi^{*}\right]} F\left[\Psi, \Psi^{*}\right]}{\int D \Psi D \Psi^{*} e^{-S_{(J)}\left[\Psi, \Psi^{*}\right]}},
$$

is invariant under chiral transformations (101) or (104), since $D \Psi D \Psi^{*} \rightarrow$ $D \Psi D \Psi^{*}$.

\section{$5 \quad$ Summary and outlook}

In treating the topologically nontrivial complex scalar field configurations in the noncommutative case, our main tool was the noncommutative version of the Hopf fibration encoded in the noncommuting parameters $\hat{\chi}_{\alpha}, \hat{\chi}_{\alpha}^{*}, \alpha=1,2$. Any field configuration with the topological winding number $\kappa$ was expanded into the functions $\Phi_{J k m}^{j}, k=\frac{1}{2}(M-N),|m| \leq j=|k|, \ldots, \frac{1}{2}(M+N)$ where $\Phi_{J k m}^{j}$ are noncommutative analogues of the standard $D$-functions $D_{k m}^{j}$. Thus we gave an algebraic characterization of the winding number $\kappa=2 k$, which is directly related to the index $k$ of the $D$-functions in question. On the matrix level this leads to the $(M+1) \times(N+1)$-matrix representation of fields from the space $\hat{\mathcal{H}}_{M N}$. The usual matrix geometry models correspond to $M=N$, and this is the reason why they describe the topologically trivial configurations only.

The same procedure applied for the treatment of the topologically nontrivial spinor field configurations, too. Moreover, here the natural supersymmetry of the problem introduced in [6] was essential; we described the topologically nontrivial spinors on $S^{2}$ as the odd sections of nontrivial super-line 
bundles on the supersphere $s S^{2}$. For this purpose we used the noncommutative Hopf superfibration in terms of even noncommutative parameters $\hat{\chi}_{\alpha}$, $\hat{\chi}_{\alpha}^{*}, \alpha=1,2$, to which we added a pair $\hat{a}, \hat{a}^{*}$ of odd noncommutative parameters. We identified the spinor bundle $\hat{\mathcal{S}}_{M N}$ as the smallest space that is invariant under action of the Dirac operator $D$. The bundle $\hat{\mathcal{S}}_{M N}$ is spanned by $|M-N|$ zero modes of $D$ and by the functions $\Phi_{J-\frac{1}{2}, k-\frac{1}{2}, m}^{j} \hat{a}^{*}, \Phi_{J-\frac{1}{2}, k+\frac{1}{2}, m}^{j} \hat{a}$ corresponding to the non-zero modes of $D$.

The models (98) and (100) are rotationally invariant and contain only a finite number of degrees of freedom on both classical and quantum levels. This truncation of the modes has the consequence that their quantum version is UV-regular (there are only finite sums instead of singular integrals and/or infinite series). A detailed discussion of these aspects in the case of a real scalar field can be found in [5].

The supersymmetry approach proposed in [6] proved to be useful in describing the chiral properties of spinors. Our spinor-field models have chirally invariant actions; however, the field functional measure $\left[D \Psi, D \Psi^{*}\right]_{M N}$ is not invariant for a fixed $M-N \neq 0$ and, under chiral transformations, it is modified due to the zero modes by the factor $e^{i \kappa \alpha}$. Only when the fields with given $\kappa$ and $-\kappa$ are treated simultaneously is the chiral invariance recovered. Thus the chiral properties of the theory are the same as those in the standard untruncated case.

It would be desirable to include the gauge fields into our approach. Even more desirable and important is to extend our scheme to the four-dimensional (super)sphere $S^{4}$. We hope to attack these problems in a near future.

Acknowledgements We are grateful to A. Connes, V. Černý, T. Damour, M. Fecko, J. Fröhlich, J. Ftáčnik, K. Gawȩdzki, J. Madore, H. Neuberger and D. Sullivan for useful discussions. 


\section{References}

[1] A. Connes, Publ. IHES 62 (1986) 257.

[2] A. Connes, Noncommutative Geometry (Academic Press, London, 1994).

[3] M. Dubois-Violette, C. R. Acad. Sci. Paris 307, Ser. I (1988) 403.

[4] M. Dubois-Violette, R. Kerner and J. Madore, J. Math. Phys. 31 (1990) 316.

[5] H. Grosse, C. Klimčík and P. Prešnajder, Towards finite quantum field theory in non-commutative geometry, preprint CERN-TH/95-138, UWThPh-19-1995, to appear in Int. J. Mod. Phys. A.

[6] H. Grosse, C. Klimčík and P. Prešnajder, Field theory on a supersymmetric lattice, preprint CERN-TH/95-195, UWThPh-20-1995.

[7] F.A. Berezin, Commun. Math. Phys. 40 (1975) 153.

[8] J. Hoppe, MIT PhD Thesis, 1982 and Elem. Part. Res. J. (Kyoto) 80 (1989) 145.

[9] J. Madore, J. Math. Phys. 32 (1991) 332 and Class. Quant. Grav. 9 (1992) 69.

[10] H. Grosse and P. Prešnajder, Lett. Math. Phys. 33 (1995) 171.

[11] H. Grosse and J. Madore, Phys. Lett. B283 (1992) 218.

[12] C. Jayewardena, Helv. Phys. Acta 61 (1988) 636.

[13] H. Neuberger, private communication.

[14] A. P. Prudnikov, Yu. A. Brytshkov and O. I. Maritshev, Integrals and Series, (Nauka, Moscow, 1981, in Russian).

[15] M. Scheunert, W. Nahm and V. Rittenberg, J. Math. Phys. 18 (1977) 146 , and 154. 